\title{
The Prevalence of Dental Anxiety Associated with Pain among Chinese Adult Patients in Guangzhou
}

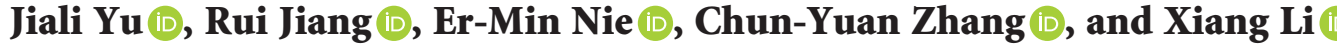 \\ First Affiliated Hospital, Sun Yat-Sen University, 58 Zhongshan 2nd Road, Guangzhou 510080, China \\ Correspondence should be addressed to Rui Jiang; jiangr9@mail.sysu.edu.cn
}

Received 6 April 2021; Revised 27 May 2021; Accepted 8 June 2021; Published 14 June 2021

Academic Editor: Mohammad Khan

Copyright @ 2021 Jiali Yu et al. This is an open access article distributed under the Creative Commons Attribution License, which permits unrestricted use, distribution, and reproduction in any medium, provided the original work is properly cited.

\begin{abstract}
Background. Anxious people appear to exaggerate the severity of aversive experiences such as anxiety and pain. Anxiety towards dental procedures is a common difficulty that may be experienced by dental patients all over the world. The goal of the study is to find out the prevalence of dental anxiety and its associated factors in Chinese adult patients. Methods. A cross-sectional study was conducted on 183 dental adult patients whose age ranged from 18 to 70 years. Demographic details, first and most recent dental visits with experience, the MDAS, and the Visual Analogue Scale for Anxiety (VAS-A) were obtained. Data were analyzed by frequency analysis, chi-square test, and Spearman correlation test. Results. Most of the respondents were female (68.9\%) and $30-45$ years age group. The mean total score for dental anxiety on the MDAS was 13.63 (3.1). 80.3\% of participants suffered from moderate or high dental anxiety. Age must show a strong association with dental anxiety among the participants $(p=0.011)$. The first dental visit experience, the frequency of the dental visit, most recent dental experience, length of time since the most recent dental visit, and postponement of the dental visit are strongly associated with the MDAS score $(p=0.001)$. Conclusions. The MDAS score exhibits that Chinese adult patients have significant dental anxiety and phobia. Identifying patients with dental anxiety as soon as possible is essential to providing better dental care.
\end{abstract}

\section{Introduction}

Dental anxiety is a distress and unease situation that occurred in patients against the dental treatment procedure [1]. Despite modern and technical advancements in dentistry, existing research shows that dental anxiety remains a concern. Amid modern dental innovation and technical advancements, dental anxiety continues to be a major issue affecting both children and adults. Several studies have found a "dynamic vicious cycle" linking dental anxiety to poor oral health $[2,3]$. Dental anxiety comprises a large proportion of people of all ages and social backgrounds, which often leads to poor oral health due to total avoidance of dental care, inconsistent dental attendance, or poor engagement [4]. Patients who suffer from dental anxiety are more likely to postpone or neglect dental treatment, resulting in the deterioration of their oral health. The progression of untreated oral infections, combined with feelings of remorse, humiliation, or worthlessness, contributes to an increase in dental anxiety, and the vicious cycle continues [3]. According to a recent study, 83.1 percent of Chinese adult patients had moderate to severe dental anxiety, and 16.2 percent met the requirements for specific dental phobia [5]. Patients suffering from dental anxiety are a significant source of stress that can jeopardize the dentist's clinical efficiency. As a result, patients with dental anxiety must be identified before treatment initiation. It will assist dental care professionals in breaking the cycle and providing successful treatment [6].

The Modified Dental Anxiety Scale (MDAS) is a reliable and factual tool used in clinical settings to assess dental anxiety [7]. MDAS is an extension of Corah's Dental Anxiety Scale (CDAS), a 4-item tool that asks patients to quantify their anxiety levels in four different dental circumstances. Humphris et al. suggested that MDAS improves on the original CDAS by including a question item about receiving local anesthetic injections and requesting the potential answers to each question on a Likert scale ranging from "not 
TABLE 1: Characteristics of the study participants.

\begin{tabular}{|c|c|c|c|}
\hline Variables & & Frequency & Percentage (\%) \\
\hline \multirow{2}{*}{ Gender } & Male & 57 & 31.1 \\
\hline & Female & 126 & 68.9 \\
\hline \multirow{4}{*}{ Age (years) } & $18-30$ & 57 & 31.1 \\
\hline & $30-45$ & 66 & 36.1 \\
\hline & $46-60$ & 48 & 26.2 \\
\hline & $60-70+$ & 12 & 6.6 \\
\hline \multirow{4}{*}{ Educational level } & Uneducated & 36 & 19.7 \\
\hline & High school & 67 & 36.6 \\
\hline & Degree/diploma & 59 & 32.2 \\
\hline & Postgraduation & 21 & 11.5 \\
\hline \multirow{4}{*}{ Employment } & Employed (full time) & 87 & 47.5 \\
\hline & Unemployed & 24 & 13.1 \\
\hline & Student & 42 & 23.0 \\
\hline & Retired & 30 & 16.4 \\
\hline \multirow{3}{*}{ Self-perceived oral health } & Good & 32 & 17.5 \\
\hline & Average & 101 & 55.2 \\
\hline & Poor & 50 & 27.3 \\
\hline \multirow{2}{*}{ Dental experience } & Yes & 114 & 62.3 \\
\hline & No & 69 & 37.7 \\
\hline \multirow{3}{*}{ First dental visit } & $<12$ years old & 43 & 23.5 \\
\hline & $12-18$ years old & 82 & 44.8 \\
\hline & $>18$ years old & 58 & 31.7 \\
\hline \multirow{3}{*}{ First dental experience } & Good & 56 & 30.6 \\
\hline & Not bad & 77 & 42.1 \\
\hline & $\mathrm{Bad}$ & 50 & 27.3 \\
\hline \multirow{3}{*}{ Frequency of dental visits } & Every 6 months & 72 & 39.3 \\
\hline & Every 12 months & 49 & 26.8 \\
\hline & Less frequent/when needed & 62 & 33.9 \\
\hline \multirow{2}{*}{ Most recent dental experience } & Good & 108 & 59.0 \\
\hline & $\mathrm{Bad}$ & 75 & 41.0 \\
\hline \multirow{3}{*}{ The length of time since the most recent dental visit } & Within 3 months & 82 & 44.8 \\
\hline & 3-12 months & 54 & 29.5 \\
\hline & Longer than 12 months & 47 & 25.7 \\
\hline \multirow{2}{*}{ Postponement of the dental visit } & Yes & 110 & 60.1 \\
\hline & No & 73 & 39.9 \\
\hline MDAS score & & \multicolumn{2}{|c|}{$13.63(3.1)^{*}$} \\
\hline \multirow{3}{*}{ MDAS score level } & Less & 36 & 19.7 \\
\hline & Moderate & 128 & 69.9 \\
\hline & Severe & 19 & 10.4 \\
\hline
\end{tabular}

${ }^{*}$ Mean (SD), descriptive and frequency analyses.

anxious" to "extremely anxious." [7] The MDAS has the benefit of being a cost-effective tool for population-based research due to its simplicity [8]. Dental anxiety and fear have a detrimental effect on oral and dental health these days. It also causes complications and increases in costs. Based on the existing literature findings, we aimed to investigate the prevalence of dental anxiety and the factors that could induce dental anxiety in Chinese adult patients who visited our dental clinic at Guangzhou. We assume that the findings can also be used to minimize dental anxiety.

\section{Methods}

183 Chinese adult patients in First Affiliated Hospital, Sun Yat-Sen University, were included in this study according to inclusion criteria. Each participant received written informed consent and signed before participating to this study.

Each participant was given a set of questionnaires adopted from Dou et al. including three sections in this cross-sectional study [5]. In the first section, the question was regarding sociodemographics, oral health practice, and dental visits. Assessment of dental anxiety was done in the second section of the questionnaire. A Chinese version of the Modified Dental Anxiety Scale (MDAS) was adopted from Dou et al. [5] and divided into a 5-item measure to assess fear of dental procedures, including before going for treatment, waiting for treatment, drilling, cleaning, and local anesthetic injections. Ratings of 1-5 indicated "not anxious," "slightly anxious," "fairly anxious," "very anxious," and "extremely anxious" correspondingly. Dental fear can be described 
TABLE 2: Participant responses for the MDAS.

\begin{tabular}{|c|c|c|c|c|c|}
\hline Item name & Not anxious & Slightly anxious & Fairly anxious & Very anxious & Extremely anxious \\
\hline Before going for treatment & $73(39.9 \%)$ & $37(20.2 \%)$ & $55(30.1 \%)$ & $18(9.8 \%)$ & $0(0 \%)$ \\
\hline Waiting for treatment & $90(49.2 \%)$ & $19(10.4 \%)$ & $74(40.4 \%)$ & $0(0 \%)$ & $0(0 \%)$ \\
\hline Drilling & $19(10.4 \%)$ & $36(19.7 \%)$ & $18(9.8 \%)$ & $110(60.1 \%)$ & $0(0 \%)$ \\
\hline Cleaning & $36(19.7 \%)$ & $72(39.3 \%)$ & $56(30.6 \%)$ & $0(0 \%)$ & $19(10.4 \%)$ \\
\hline Local anesthetic injections & $37(20.2 \%)$ & $0(0 \%)$ & $18(9.8 \%)$ & $36(19.7 \%)$ & $92(50.3 \%)$ \\
\hline
\end{tabular}

Frequency analysis.

TABLe 3: Association of the MDAS score and other risk factors.

\begin{tabular}{lcc}
\hline Variables & $X^{2}(\mathrm{df})$ & $p$ value \\
\hline Gender & $6.092(6)$ & 0.413 \\
Age & $34.626(18)$ & 0.011 \\
Educational level & $16.742(18)$ & 0.541 \\
Employment & $10.373(18)$ & 0.919 \\
Self-perceived oral health & $3.827(12)$ & 0.986 \\
Dental experience & $1.591(6)$ & 0.953 \\
First dental visit & $25.672(12)$ & 0.012 \\
First dental experience & $0.846(120)$ & 0.999 \\
Frequency of dental visits & $127.511(12)$ & 0.001 \\
Most recent dental experience & $61.196(6)$ & 0.001 \\
The length of time since the most recent dental visit & $110.845(12)$ & 0.001 \\
Postponement of the dental visit & $44.281(6)$ & 0.001 \\
\hline
\end{tabular}

Chi-square (degree of freedom).

when the total MDAS score was 19 or above [4]. The researchers were trained and calibrated before this study, and the reliability of the intraexaminer was evaluated using the kappa test. A kappa value of 0.780 was achieved. Visual Analogue Scale (VAS) was used in the third section to assess the pain level for dental experiences.

All statistical analyses were performed by IBM SPSS v. 26. The mean total MDAS score was calculated for all the categorized variables. Descriptive and frequency analyses were performed on all variables. A chi-square test was performed to compare the mean MDAS score between categories in the same variable. Tukey's post hoc test was performed to control for multiple comparisons. Spearman rank correlation was done to assess the strength of association between MDAS and variables.

\section{Results}

Table 1 shows the characteristics of the study participants. Among the 183 respondents, $31.1 \%$ were males and $68.9 \%$ were females. Most of the patients were 30-45 years age group $(36.1 \%)$. The mean total score for dental anxiety on the MDAS was 13.63 (3.1). Based on the MDAS score, $19.7 \%$ of the subjects were identified to be less anxious (5-9 total score), $69.9 \%$ were moderately anxious (10-18 total score), and $10.4 \%$ were seriously anxious ( $\geq 19$ total score). Most of the participants had an average level of self-perceived oral health (55.2\%). Most of them went to the dentist for the first time when they were $12-18$ years old $(44.8 \%)$ with not bad (42.1\%) to bad experiences (27.3\%). Among the respondents, 75 had reported bad experiences in their most recent dental visit. More than half of the participants (60.1\%) had reported that they postponed their dental visit.

Table 2 shows the participant responses for each MDAS item. Before going to treatment, 39.9\% did not feel anxious, while $30.1 \%$ were anxious. Nearly half of the participants (49.2\%) reported that they do not feel anxious during waiting for treatment following others who felt fairly anxious (40.4\%). More than half of the participants reported that they feel very anxious during drilling (60.1\%). Half of the respondents $(50.3 \%)$ reported that they were extremely anxious about local anesthetic injections following very anxious (19.7\%).

Table 3 shows the chi-square result of the MDAS score and other variable associations. Age must show a strong association with dental anxiety among the participants $(p=0.011)$. The first dental visit is also associated with developing dental anxiety $(p=0.012)$. Having a bad experience during the first dental visit was a contributing factor for anxiety. Frequency of the dental visit, most recent dental experience, length of time since the most recent dental visit, and postponement of the dental visit are strongly associated with the MDAS score $(p=0.001)$.

Table 4 shows the correlation between the MDAS score and other variables. No correlation was found between the MDAS and variables including age, gender, educational background, employment status, and self-perceived oral health status. Negative dental experience during treatment demonstrated a strong relation with dental anxiety. Participants who had experiences of the less frequent dental visit were more anxious compared to those with more frequent visits $(p=0.001)$. Pain at the most recent dental visit or 
TABLE 4: The correlation between the MDAS score and other variables.

\begin{tabular}{lcc}
\hline Variables & Spearman correlation & $p$ value \\
\hline Gender & -0.085 & 0.324 \\
Age & -0.034 & 0.644 \\
Educational level & -0.096 & 0.197 \\
Employment & -0.009 & 0.909 \\
Self-perceived oral health & 0.061 & 0.415 \\
Dental experience & -0.019 & 0.804 \\
First dental visit & -0.080 & 0.282 \\
First dental experience & 0.010 & 0.892 \\
Frequency of dental visits & -0.337 & 0.001 \\
Most recent dental experience & -0.064 & 0.393 \\
The length of time since the most recent dental visit & -0.062 & 0.43 \\
Postponement of the dental visit & 0.033 & 0.653 \\
\hline
\end{tabular}

Chi-square (degree of freedom).

before the present dental visit was an important factor correlating with dental anxiety among participants.

\section{Discussion}

"Dental anxiety" and "dental phobia" create a major problem for both patients and dentists. These two words are often used synonymously; however, there are key differences between them. Dental anxiety is characterized as a specific patient response to dental procedure-related stress when the trigger is unclear, ambiguous, or not present at the time [9]. Dental phobia is described as intense and constant anxiety in a dental setting, which causes the person to avoid going to the dentist at all costs unless a physical issue becomes overwhelming [10]. It is recommended that dental practitioners evaluate dental anxiety and dental phobia during clinical assessments using a valid and reliable scale that can accurately measure the subjective experience of dental anxiety and phobia [10].

The results of the present study indicate an increase in dental anxiety among patients with pulpal pain as compared to previous studies $[10,11]$. Our findings have shown that young patients were more likely to experience dental anxiety and phobia than older patients. Furthermore, past negative experiences in childhood and adolescence, periodontal problem perception, and feeling the gag reflex during dental care were risk factors for dental anxiety and dental phobia. In our study, $83.3 \%$ of the subjects reported moderate to extreme dental anxiety, which is higher than the results of previous studies. Another Chinese study by Duo et al. found that $83.1 \%$ of people had moderate to severe dental anxiety [5]. According to Esmaeili et al.'s [12] Brazilian report, more than half of the patients (60.4\%) were moderately or highly anxious. Different studies also show different prevalence rates of dental anxiety. The dental phobia criteria (MDAS score $>19$ ) were met by $10.2 \%$ of participants in our study, which was comparatively higher than many other previous studies $[13,14]$. The findings' incompatibility can be due to variations in the cultural background and the dental features of the patients in the above study.

The most important predictors of dental anxiety and phobia were age and a past negative experience in a dental clinic. Many studies have shown that females are more afraid of dental treatment than males $[14,15]$. Other related research, however, found no gender differences in dental anxiety $[16,17]$. In our study, no significant differences in dental anxiety were found between men and women, supporting the results of previous studies conducted in Tanzania and Nepal $[16,17]$. The relationship between age and the level of dental anxiety is still uncertain in the literature, and researchers have suggested conflicting findings. In the current study, the effect of age on the MDAS score was significant, with older patients scoring significantly lower than younger patients. This finding is consistent with the findings of many other related studies $[9,14,16,18]$. Patients seeking pain relief were always fearful of the pain they would experience during the assessment and treatment procedure. Invasive procedures include local anesthetic injection, drilling the tooth, and removing the dental pulp [19]. Pain is a significant factor in dental anxiety. According to the literature, the primary cause of dental phobia is anxiety regarding pain during dental treatment [20]. The effect of a previous bad experience in a dental clinic on the MDAS score was also significant, with patients who had a previous bad experience in their first dental visit or most recent dental visit scoring substantially higher than patients who had no such experience. This result is completely consistent with the results of other studies [21-23]. A significant difference was found in terms of the frequency of visits to a dentist and length of time since the recent dental visit. Other researchers have reported similar results, claiming that patients who visit the dentist daily are less likely to exhibit dental anxiety [15].

The current study's findings must be interpreted considering many methodological limitations. Because of the convenience sampling technique used to choose patients and the lack of randomization, there is a risk of selection bias. Within the limitation of this research, it should be noted that this is a cross-sectional study, which makes it impossible to analyse causal relationships. Dental practitioners play an important role in the treatment and prevention of dental anxiety. It has been reported that people who suffer from dental anxiety can have a detrimental impact on their tooth examination and oral health [11]. We can avoid problems by asking the factors that can cause distress in patients before dental examinations, and we can estimate the risk to people's oral health. Future research is recommended to allow for 
further investigation and confirmation of the study's findings. A prospective study will support both dentists and patients, with the potential to address the drawbacks of a cross-sectional study.

\section{Conclusion}

Dental anxiety is common among Chinese adult patients. The results of this study support the hypothesis that people who are predisposed to fearfully react to pain are more likely to become stuck in a vicious cycle of anxiety, fear of pain, and avoidance of dental care. Younger age people, first time dental treatment seekers, and having previous bad experience are the main risk factors for dental anxiety found in our study. Addressing these aspects can enhance the efficiency of strategies for reducing anxiety and phobia in adult dental patients.

\section{Data Availability}

The data used to support the findings of this study are available from the corresponding author upon request.

\section{Conflicts of Interest}

The authors declare that there are no conflicts of interest regarding the publication of this paper.

\section{Authors' Contributions}

Rui Jiang participated in the drafting, data collection, data analysis, description of results, discussion, critical revision of the article, and evaluation of the final version of the manuscript. Jiali $\mathrm{Yu}$ participated in the drafting, data collection, data analysis, description of results, and discussion. Er-Min Nie participated in the drafting, data analysis, and description of results. Xiang $\mathrm{Li}$ and Chun-Yuan Zhang participated in the description of results and discussion.

\section{Acknowledgments}

The authors are grateful to all participants who willingly participated in their study. They are also thankful to their biostatistics department. This research was supported by First Affiliated Hospital, Sun Yat-sen University, Guangzhou, China.

\section{References}

[1] B. Yakar, T. Ö Kaygusuz, and E. Pirinçci, "Evaluation of dental anxiety and fear in patients who admitted to the faculty of dentistry: which patients are riskier in terms of dental anxiety," Ethiopian Journal of Health Sciences, vol. 29, no. 6, pp. 719-726, 2019.

[2] H. Kassem El Hajj, Y. Fares, and L. Abou-Abbas, "Assessment of dental anxiety and dental phobia among adults in Lebanon," BMC Oral Health, vol. 21, no. 1, pp. 1-10, 2021.

[3] J. M. Armfield, "What goes around comes around: revisiting the hypothesized vicious cycle of dental fear and avoidance," Community Dentistry and Oral Epidemiology, vol. 41, no. 3, pp. 279-287, 2013.
[4] E. P. Tunc, D. Firat, O. D. Onur, and V. Sar, "Reliability and validity of the modified dental anxiety scale (MDAS) in a Turkish population," Community Dentistry and Oral Epidemiology, vol. 33, no. 5, pp. 357-362, 2005.

[5] L. Dou, M. M. Vanschaayk, Y. Zhang, X. Fu, P. Ji, and D. Yang, "The prevalence of dental anxiety and its association with pain and other variables among adult patients with irreversible pulpitis," BMC Oral Health, vol. 18, no. 1, pp. 1-6, 2018.

[6] A. Crego, M. Carrillo-Díaz, J. M. Armfield, and M. Romero, "From public mental health to community oral health: the impact of dental anxiety and fear on dental status," Frontiers in Public Health, vol. 2, p. 16, 2014.

[7] G. M. Humphris, T. Morrison, and S. J. Lindsay, "The modified dental anxiety scale: validation and United Kingdom norms," Community Dental Health, vol. 12, no. 3, pp. 143-150, 1995.

[8] S. Cohen, J. Fiske, and J. Newton, "The impact of dental anxiety on daily living," British Dental Journal, vol. 189, no. 7, pp. 385-390, 2000.

[9] G. M. Humphris, T. A. Dyer, and P. G. Robinson, "The modified dental anxiety scale: UK general public population norms in 2008 with further psychometrics and effects of age," BMC Oral Health, vol. 9, no. 1, pp. 20-28, 2009.

[10] A. N. Asl, M. Shokravi, Z. Jamali, and S. Shirazi, "Barriers and drawbacks of the assessment of dental fear, dental anxiety and dental phobia in children: a critical literature review," Journal of Clinical Pediatric Dentistry, vol. 41, no. 6, pp. 399-423, 2017.

[11] D. Appukuttan, S. Subramanian, A. Tadepalli, and L. Damodaran, "Dental anxiety among adults: an epidemiological study in South India," North American Journal of Medical Sciences, vol. 7, no. 1, pp. 13-18, 2015.

[12] H. Esmaeili, M. Malekzadeh, D. Esmaeili, and F. Nikeghbal, "Dental anxiety and the effectiveness of local anesthesia," Brazilian Journal of Oral Sciences, vol. 19, Article ID e, 2020.

[13] K. Strøm, A. B. Skaare, and T. Willumsen, "Dental anxiety in 18 year-old Norwegians in 1996 and 2016," Acta Odontologica Scandinavica, vol. 78, no. 1, pp. 13-19, 2020.

[14] A. M. White, L. Giblin, and L. D. Boyd, "The prevalence of dental anxiety in dental practice settings," Journal of Dental Hygiene: JDH, vol. 91, no. 1, pp. 30-34, 2017.

[15] M. Saatchi, M. Abtahi, G. Mohammadi, M. Mirdamadi, and E. S. Binandeh, "The prevalence of dental anxiety and fear in patients referred to Isfahan dental school, Iran," Dental Research Journal, vol. 12, no. 3, pp. 248-253, 2015.

[16] I. K. Minja, A. C. Jovin, and G. J. Mandari, "Prevalence and factors associated with dental anxiety among primary school teachers in Ngara district, Tanzania," Tanzania Journal of Health Research, vol. 18, no. 1, pp. 1-10, 2016.

[17] J. Giri, P. R. Pokharel, R. Gyawali, and B. Bhattarai, "Translation and validation of modified dental anxiety scale: the Nepali version," International Scholarly Research Notices, vol. 2017, Article ID 5495643, 5 pages, 2017.

[18] W. K. Leung, Y. R. Duan, X. X. Dong et al., "Treatment of periodontal diseases," Economic Periodontal and Implant Dentistry, vol. 14, pp. 165-239, 2016.

[19] A. W. Wong, C. S. Tsang, S. Zhang, K. Y. Li, C. Zhang, and C. H. Chu, "Treatment outcomes of single-visit versus multiple-visit non-surgical endodontic therapy: a randomised clinical trial," BMC Oral Health, vol. 15, no. 1, p. 162, 2015.

[20] A. E. Carter, G. Carter, M. Boschen, E. AlShwaimi, and R. George, "Pathways of fear and anxiety in dentistry: a 
review," World Journal of Clinical Cases, vol. 2, no. 11, p. 642, 2014.

[21] M. L. Goettems, T. M. Ardenghi, F. F. Demarco, A. R. Romano, and D. D. Torriani, "Children's use of dental services: influence of maternal dental anxiety, attendance pattern, and perception of children's quality of life," Community Dentistry and Oral Epidemiology, vol. 40, no. 5, pp. 451-458, 2012.

[22] E. Nicolas, V. Collado, D. Faulks, B. Bullier, and M. Hennequin, "A national cross-sectional survey of dental anxiety in the French adult population," BMC Oral Health, vol. 7, no. 1, pp. 12-17, 2007.

[23] C. W. Lian, T. S. Phin, C. S. Chat, B. C. Shin, L. H. Baharuddin, and Z. B. Jalil, "Oral health knowledge, attitude and practice among secondary school students in Kuching, Sarawak," Archives of Orofacial Sciences, vol. 5, no. 1, pp. 9-16, 2010. 